\title{
UTILIZAÇÃO DE AGENTE ESCLEROSANTE NO TRATAMENTO DE HEMANGIOMA LINGUAL: RELATO DE CASO
}

\section{USE OF SCLEROSING AGENT IN THE TREATMENT OF LINGUAL HEMANGIOMA: A CASE REPORT}

\author{
Pedro Henrique Da Hora Sales* \\ Raquel Bastos Vasconcelos** \\ Anderson Maia Meneses"** \\ Daniel Facó da Silveira Santos ${ }^{* * * *}$
}

\section{RESUMO}

O hemangioma é uma neoplasia benigna que se caracteriza por proliferação endotelial anormal. Acomete a região maxilofacial com predileção pelos lábios, língua e mucosa jugal. O tratamento pode ser realizado por diferentes formas terapêuticas, desde corticoterapia até a excisão cirúrgica. Neste trabalho, descrevemos um caso clínico de hemangioma lingual, no qual se optou pela utilização de oleato de monoetanolamina a 5\% ethamolin $^{\circledR}$, obtendo-se total involução da lesão em 3 aplicações, além de nenhum sinal de recidiva após 15 meses.

Descritores: Hemangioma • Patologia bucal • Anormalidades da boca.

\section{ABSTRACT}

The hemangioma is a benign neoplasm characterized by abnormal endothelial proliferation. It affects the maxillofacial region with predilection for the lips, tongue and buccal mucosa. The treatment can be performed by different therapeutics, since steroids to surgical excision. In this work we describe a case of lingual hemangioma, where we opted to use monoethanolamine oleate $5 \%$ - ethamolin ${ }^{\circledR}$ to get the total lesion regression in 3 applications, and no sign of recurrence after 15 months.

Descriptors: Hemangioma • Pathology, oral • Mouth abnormalities

\footnotetext{
* Cirurgião-dentista, residente de cirurgia bucomaxilofacial do hospital Instituto Dr. José Frota - Fortaleza-CE - salespedro@gmail.com ** Cirurgiã-dentista, residente de cirurgia bucomaxilofacial do Hospital Batista Memorial - Fortaleza-CE - raquelbastosvasconcelos@hotmail.com *** Cirurgião-dentista, residente de cirurgia bucomaxilofacial do Hospital Geral de Fortaleza SESA - Fortaleza-CE - andersonmmeneses@gmail.com **** Cirurgião bucomaxilofacial especialista pelo Colégio Brasileiro de Cirurgia e Traumatologia Bucomaxilofacial. Preceptor da residência em Cirurgia Bucomaxilofacial do Hospital Batista Memorial, Fortaleza -CE e Hospital Geral de Fortaleza SESA - Fortaleza-CE. danielsilveirasantos@gmail.com
} 
SALES PHDH

VASCONCELOS RB

MENESES AM

SANTOS DFS

UT I LIZAÇÃO

DE AGENTE

ESCLEROSANTE

NO TRATAMENTO

DE HEMANGIOMA

LINGUAL:

RELATO DE CASO

\section{$254 \ldots$}

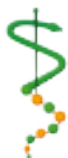

REV, ODONTOL,

UNIV. CID. SÃO

PAULO

$2015 ; 27(3): 253-$

7, SET-DEZ :

\section{N T R O D U ÇÃ O}

O hemangioma é considerado pela Organização Mundial de Saúde (OMS) uma neoplasia benigna vascular, cuja principal característica é a proliferação de células endoteliais ${ }^{1}$. Essa lesão normalmente é encontrada na infância, de forma congênita, embora possa desenvolver-se em adultos, tem predileção pelo gênero feminino e por indivíduos leucodermas ${ }^{2-4}$.

De uma forma geral, o hemangioma apresenta-se como lesão única, podendo ser observado em qualquer parte do organismo, contudo é mais incidente na área de cabeça e pescoço com predominância nos lábios, língua e mucosa jugal 2,3,5.

Clinicamente, o hemangioma pode apresentar-se de diversas formas, sendo observado, geralmente, em forma de boIha preenchida completamente por conteúdo sanguíneo, desencadeando uma coloração azul-violácea que empalidece e diminui de tamanho à compressão ${ }^{3,6-8}$. Essa compressão consiste em uma manobra semiotécnica denominada de diascopia, contudo, em caso de dúvida quanto ao calibre vascular que nutre a lesão, recomenda-se a arteriografia ou ecografia com doppler colorido, ressonância magnética e tomografia computadorizada $6,7,9$.

O hemangioma pode ser classificado histologicamente em capilar ou cavernoso e, apesar de existirem outras classes de hemangioma, estas serão variações dos dois tipos básicos ${ }^{6,8,10}$.

O tratamento do hemangioma pode ser realizado por diferentes formas terapêuticas conforme a localização, extensão e etiologia da lesão. Na literatura observa-se, em casos congênitos, o uso da proservação devido à possibilidade de total remissão com a idade, contudo, se a involução espontânea não for uma opção, pode-se realizar infiltração de solução química esclerosante, crioterapia, laserterapia, corticoterapia e embolia vascular, cada uma isoladamente ou de forma combinada com a excisão cirúrgica ${ }^{8,9,11,12}$.

Dentre as possíveis terapêuticas existentes, a esclerose por infiltração de agente químico é uma das modalidades terapêuticas mais utilizadas, uma vez que tem como vantagem ser um procedimento pouco invasivo e não promover cicatriz aparente. Apresenta poucas complicações, porém traz a desvantagem de não poder ser utilizada em lesões de grandes dimensões, pois o fluxo sanguíneo rápido e os espaços vasculares largos e difundidos acabam por diluir e proporcionar um pequeno contato com as paredes vasculares, ocasionando efeito mínimo ou ausente do agente químico com o endotélio vascular ${ }^{8,11,13}$

Entre as substâncias químicas possíveis de serem utilizadas para a infiltração, o oleato de monoetanolamina (OM) a 5\% - ethamolin ${ }^{\circledR}$ apresenta-se como agente mais utilizado devido a sua menor toxicidade $^{12-15}$.

No hemangioma o OM a 5\% atua promovendo irritação da camada íntima do endotélio vascular, produzindo uma resposta inflamatória estéril e consequente fibrose da parede do vaso ocasionando posterior redução e possível oclusão da luz vascular ${ }^{10,11,12}$.

Esse agente químico utilizado na escleroterapia pode desencadear uma sensação de ardor durante sua infiltração, assim como a formação de úlcera ou necrose superficial do tecido envolvido. Entretanto, visando minimizar tais desconfortos, pode-se fazer uso de anestesia local antes de sua aplicação, além de se depositar a substância em uma região mais profun$\mathrm{da}^{10,12,14}$.

A quantidade e o intervalo da administração do OM a 5\% devem ser proporcionais ao tamanho da lesão, preconizando-se, de modo geral, que sua aplicação seja realizada em sessões intercaladas com no mínimo sete dias entre cada aplicação, não ultrapassando $2 \mathrm{ml}$ por infiltração ${ }^{10,14}$.

Tendo em vista a aplicabilidade dessa substância como alternativa terapêutica minimamente invasiva, este trabalho propõe-se a apresentar a resposta clínica de um hemangioma lingual em paciente adulto do gênero masculino perante o OM a 5\%.

\section{RELATO DE CASO}

O paciente T.C.G.D.N., do gênero masculino, leucoderma, 23 anos de idade, normossistêmico, compareceu ao ambulatório do serviço de cabeça e pescoço de um hospital público de Fortaleza do Estado 
do Ceará, queixando-se de um aumento de volume em região de borda lateral de língua, percebido cerca de 4 meses antes (Figura 1). Com diagnóstico sugestivo de hemangioma, o paciente foi encaminhado
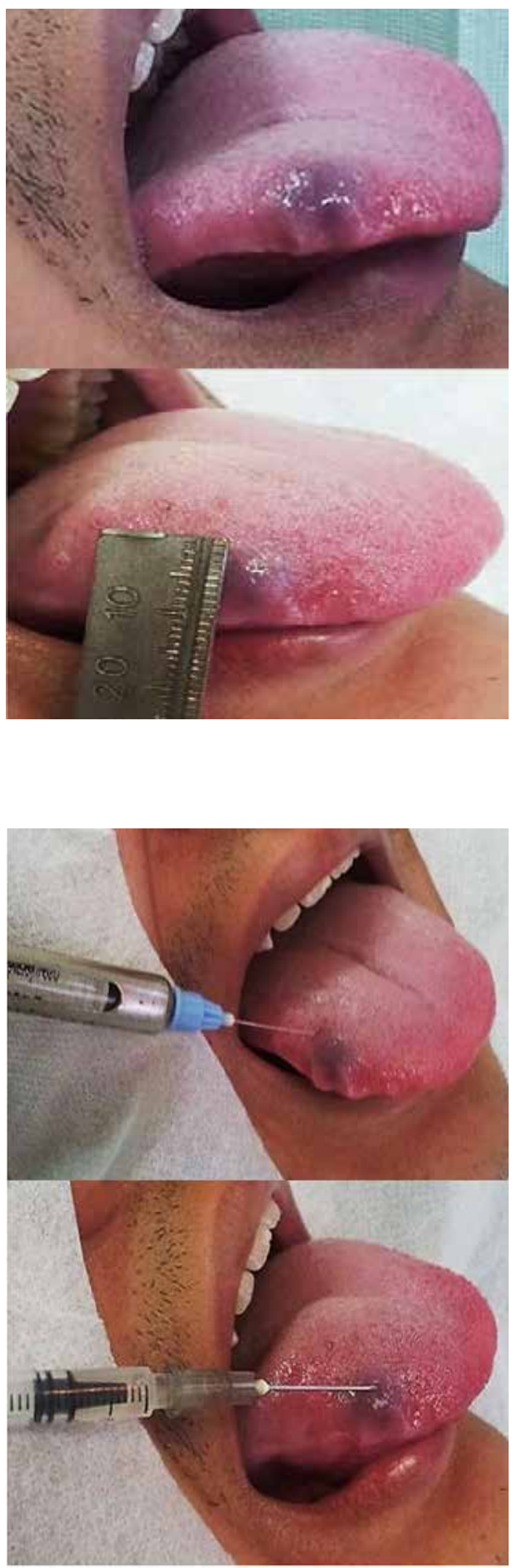

para o serviço de cirurgia e traumatologia bucomaxilofacial do setor de odontologia da Escola de Aprendizes Marinheiros do Ceará (EAMCE), a fim de confirmação do diagnóstico e possível tratamento

Na anamnese, o paciente não relatou sintomatologia dolorosa, porém informou dificuldade na mastigação, além de alteração na coloração e na forma do tecido lingual no local.

Ao exame clínico intrabucal, observou-se tumefação localizada e bem delimitada em borda lateral de língua do lado direito, com coloração azul-violácea, consistência amolecida e base séssil, medindo em média 1 x $1 \mathrm{~cm}$ no seu maior diâmetro. A manobra semiotécnica de diascopia foi realizada, verificando-se um aspecto levemente isquêmico da lesão, sugerindo uma alteração vascular do tipo hemangioma.

Sendo assim, foi solicitado um exame ecográfico (Doppler colorido) para classificar o fluxo vascular em venoso ou arterial. Após a avaliação clínica e de exames complementares, o tratamento proposto foi conservador, utilizando aplicação periódica de ethamolin ${ }^{\circledR}$ com intervalo de 10 dias entre as sessões.

Depois de realizada anestesia perilesional, com mepivacaína a $2 \%$ com epinefrina de 1:100.000, o paciente foi submetido a aplicações de $1 \mathrm{ml}$ da solução na região central e profunda da lesão. As aplicações foram realizadas com seringas de $1 \mathrm{ml}$ e agulha de insulina, através de uma injeção lenta e gradual (Figura 2). Entretanto, observou-se um discreto sangramento que foi contido com leve compressão do local.

Como resultado da primeira aplicação observou-se diminuição dimensional e alteração da coloração do hemangioma. Na segunda, houve nova regressão e aproximação da coloração normal da língua. Com a terceira aplicação, constatou-se remissão total da lesão (Figura 3).

Após 15 meses de finalizadas as aplicações, não foram observadas alterações funcionais da língua e nenhum sinal de recidiva da lesão.

\section{I S CUSSÃO}

O hemangioma é uma lesão benigna dos vasos sanguíneos, comum nos recém-nascidos, podendo, em alguns casos, se
SALES PHDH

VASCONCELOS RB

MENESES AM

SANTOS DFS

UTILIZAÇÃO

DE AGENTE

ESCLEROSANTE

NO TRATAMENTO

DE HEMANGIOMA

LINGUAL:

RELATO DE CASO

$\because 255$

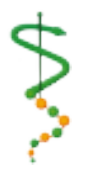

REV, ODONTOL.

UNIV. CID. SÃO

PAULO

2015; 27(3): 253-

7 , SET-DEZ 
SALES PHDH VASCONCELOS RB

MENESES AM

SANTOS DFS

UT I L I ZAÇÃO

DE AGENTE

ESCLEROSANTE

NO TRATAMENTO

DE HEMANGIOMA

LINGUAL:

RELATO DE CASO

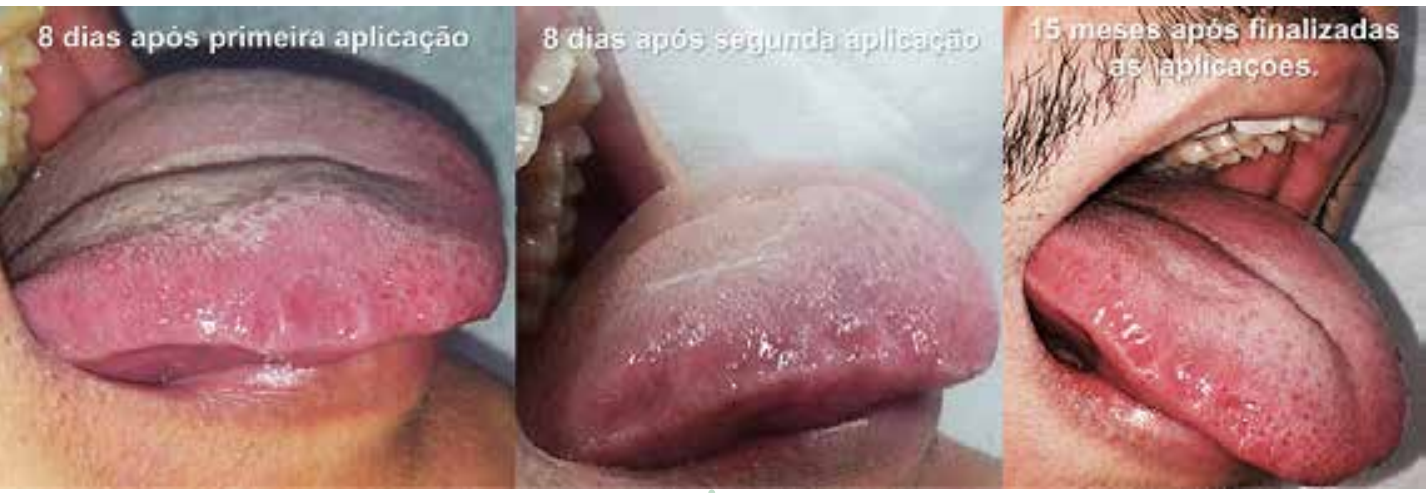

desenvolver em adultos ${ }^{3,16}$, assim como ocorreu neste relato de caso. Há uma predileção pelo gênero feminino ${ }^{2,3}$, entretanto em nosso caso, a lesão estava presente em um paciente do sexo masculino.

No que se refere à região de acometimento do hemangioma, a literatura relata uma maior incidência nos lábios seguida pela língua ${ }^{2,16}$, sendo assim confirmada por este trabalho, uma vez que a lesão estava alocada em borda lateral de língua.

Após confirmado o caráter e calibre vascular através da diascopia e da ecografia com doppler colorido, respectivamente, pôde-se planejar a terapêutica a ser aplicada e, apesar de a cirurgia ser considerada uma opção de tratamento por Choi et al. ${ }^{13} 2002$, a lesão foi tratada através da escleroterapia química, uma vez que esta promove pequena morbidade, não deixa cicatriz, evita o risco de hemorragia e é um método mais seguro e previsível ${ }^{8,11}$.

$\mathrm{O}$ agente esclerosante utilizado foi o OM a 5\% amplamente empregado na terapêutica de hemangioma com resultados satisfatórios, sendo utilizado através de infiltração intralesional, possibilitando a regressão parcial ou total da lesão ${ }^{1,3,15}$.

Em outro estudo realizado foram relatados 13 casos de hemangioma tratados por meio de escleroterapia utilizando-se OM a 5\%, em todos os pacientes; destes, foram observados que 38\% apresentaram completa regressão lesional com uma aplicação do agente esclerosante, 23\% dos casos necessitaram de duas aplicações, $31 \%$ de três aplicações e, por fim, em $8 \%$ dos casos foram necessárias qua- tro ou mais aplicações para a total regressão do hemangioma ${ }^{17}$. Em nosso caso, foram necessárias três aplicações de $1 \mathrm{ml}$ de OM a 5\% com intervalo de 10 dias entre sessões para que houvesse total regressão da lesão.

Quanto à aplicação de anestesia local prévia ao procedimento de infiltração do agente esclerosante no hemangioma, a utilização da anestesia local deve ser evitada, uma vez que, ao atingir tecidos sadios, o agente esclerosante promove sensação dolorosa, permitindo maior controle na difusão tecidual ${ }^{6}$. Por outro lado o anestésico local, através de seus agentes vasoconstrictores, assegura uma vasoconstrição periférica, limitando a ação do agente químico e aumentando seu tempo de ação nas paredes vasculares, além de evitar sintomatologia dolorosa ${ }^{18}$. No paciente do relato de caso, optou-se pela aplicação de anestésico local perilesional associado a vasoconstritor antes da aplicação do agente esclerosante, visando evitar desconforto da infiltração do agente químico.

\section{CONCLUSÃO}

O oleato de monoetanolamina a $5 \%$ apresentou-se como uma alternativa segura de menor custo financeiro e eficaz para pacientes com hemangiomas pequenos de língua. Podendo ser utilizado como uma opção terapêutica viável de menor complexidade e morbidade quando comparado à excisão cirúrgica convencional.
REV, ODONTOL, UNIV. CID. SÃO PAULO: $2015 ; 27(3): 253-$ 7 , SET-DEZ 
SALES PHDH

VASCONCELOS RB

MENESES AM

SANTOS DFS malformations with ethanolamine oleate. Asian J Surg 2008 Oct;31(4):2204. hemangioma: a report of two cases. Gen Dent 2011 May-Jun;59(3):e121-5.

2. Chinen A, Martins RH, Santos GG, Souza A, Marcucci G. Hemangioma: aspectos clínicos, diagnóstico e terapêutica de 235 casos. Rev Odontol UNICID 1996 jan-jun;8(1):43-9.

3. Neville B, Damm D, Allen C, Bouquot J. Patologia oral e maxilofacial. 3 ed. Rio de Janeiro: Elsevier; 2009.

4. Sapp J, Eversole L, Wysocki G. Patologia bucomaxilofacial contemporânea. São Paulo: Santos; 2012.

5. Gontijo B, Silva CMR, Pereira LB. Hemangioma da infância. Anais Brasileiros de Dermatologia 2003 nov-dez;78(6):651-73.

6. Rocha LB, Pádua J, Martins RH, Lia R. Hemangioma da cavidade bucal. RGO Revista Gaúcha de Odontologia 2000 48(3):150-2.

7. White S, Pharoah M. Radiologia oral fundamentos e interpretação. 5 ed. Rio de Janeiro: Elsevier; 2007.

8. Boraks S. Medicina bucal tratamento clínico-cirúrgico das doenças bucomaxilofaciais. 1 ed. São Paulo: Artes Médicas; 2011.

9. Hassani A, Khojasteh A, Vahid R. Management of mandibular vascular malformation with sclerotherapy. Report of two cases. Oral Surg Oral Med Oral Pathol Oral Radiol Endod 2006 Jul;102(1):99-103.

10. Selim H, Selim A, Khachemoune A, Metwally SA. Use of sclerosing agent in the management of oral and perioral hemangiomas: review and case reports. Med Sci Monit 2007 Sep;13(9):CS114-9.

11. Wang L, Oliveira D, Consolaro A, Perez F. Tratamento de hemangioma bucal com agente esclerosante. ROBRAC 1998 dez;7(24):20-2.
13. Choi $\mathrm{YH}, \mathrm{Han} \mathrm{MH}, \mathrm{K}$ OK, Cha $\mathrm{SH}$, Chang $\mathrm{KH}$. Craniofacial cavernous venous malformations: percutaneous sclerotherapy with use of ethanolamine oleate. J Vasc Interv Radiol 2002 May;13(5):475-82.

14. Johann AC, Aguiar MC, do Carmo MA, Gomez RS, Castro WH, Mesquita RA. Sclerotherapy of benign oral vascular lesion with ethanolamine oleate: an open clinical trial with 30 lesions. Oral Surg Oral Med Oral Pathol Oral Radiol Endod 2005 Nov;100(5):579-84.

15. Costa JR, Torriani MA, Hosni ES, D'Avila OP, de Figueiredo PJ. Sclerotherapy for vascular malformations in the oral and maxillofacial region: treatment and follow-up of 66 lesions. J Oral Maxillofac Surg 2011 Jun;69(6):e88-92.

16. Barrett AW, Speight PM. Superficial arteriovenous hemangioma of the oral cavity. Oral Surg Oral Med Oral Pathol Oral Radiol Endod 2000 Dec;90(6):731-8.

17. Prado B, Santos A, Oliveira J, Vacarrezza G, Freitas $C$. Uso de oleato de etanolamina para hemangiomas da cavidade bucal: um estudo de cinco anos. Rev Odontol Univ Cid São Paulo 2011 jan/abr;23(11):42-5.

18. Jiménez Palacios C, Pérez Herrera C, Lugo MV. La Escleroterapia como una alternativa en el Tratamiento de los Hemangiomas de los Tejidos Blandos de la CavidadBucal.ActaOdontológica Venezolana 2000 jun;38(2):4-8.

Recebido em 17/11/2015

Aceito em 08/03/2016
UTILIZAÇÃO

DE AGENTE

ESCLEROSANTE

NO TRATAMENTO

DE HEMANGIOMA

LINGUAL:

RELATO dE cASO

257
EV. ODONTOL.
NIV. CID. SÃO
AULO
O15; 27(3): $253-$ 